Analgesic Prescribing Trends in Older Veterans with Osteoarthritis: 2012-2017

\title{
Analgesic prescribing trends in a national sample of older veterans with osteoarthritis: 2012- 2017
}

Trentalange, Mark; Runels, Tessa; Bean, Andrew; Kerns, Robert D.; Bair, Matthew J.; Brody, Abraham A.; Brandt, Cynthia A.; Hwang, Ula; the EAASE (Evaluating Arthritis Analgesic Safety and Effectiveness) Investigators

\section{Introduction}

Although nationally the amounts of prescribed opioids peaked about eight years ago, opioid overdose deaths and related adverse outcomes continue to rise and have emerged as a public health emergency in the United States. $[2 ; 3 ; 5 ; 12]$ Earlier studies demonstrated increased trends in opioid prescribing and a concomitant increase in other sedative/stimulant prescription rates paralleled by adverse events, particularly hospitalizations and overdose deaths, in various populations.[11; 14; 15] Additionally, evidence from studies have started to question the effectiveness of opioids in treating some types of pain, including osteoarthritis.[7] For these reasons, national policies, guidelines and initiatives have been developed and implemented over the last several years to help clinicians decrease opioid use and to employ multiple recommended risk mitigation strategies to reduce rates of associated harms.

Recent studies have noted these initiatives to be effective with a downward trend in opioid prescribing in some settings. $[6 ; 9 ; 13 ; 16 ; 20]$ As an example, the Veterans Health Administration (VHA) launched a system-wide Opioid Safety Initiative (OSI) in October 2013 to educate prescribers about safer opioid prescribing practices, followed by a sustained organizational effort to attenuate opioid prescribing. A recent review of opioid prescription rates in the VHA system by Lin et al., 2017[9] delineated the decreasing trend in opioid prescribing since the OSI rollout.[9; 18] Investigators demonstrated changes in both rates of prescribing as well as reductions in opioid doses (quantifiable as morphine equivalents) since the initiation of the OSI.

Many studies, however, have not evaluated the impact of changing opioid prescribing practices on use of other non-opioid analgesics nor concurrent changes in reported pain intensity. Because of the continued public health threat opioids present, most studies have focused solely on opioid prescribing. $[6 ; 8]$ As policies and trends in opioid prescribing change, this will likely impact use of alternate analgesic options, especially non-opioid analgesic prescribing. These changes in practice may impact patient-reported pain intensity. Additionally, limitations of previous studies have assumed stable underlying populations, reporting total number of prescriptions by year or by month instead of calculating prescription rates. $[1 ; 9 ; 11]$ Researchers commonly have not corrected for the varying length of each month (as much as 10\%, 28-31 days) when calculating either counts or rates.

The Evaluating Arthritis Analgesic Safety and Effectiveness (EAASE) project is an ongoing multicenter observational study evaluating the safety and effectiveness of analgesic medications prescribed to older veterans who have been diagnosed with osteoarthritis (1 IO1 HX000911-01A2). Using national data collected as part of this study, we had the opportunity to evaluate national trends in opioid and nonopioid analgesic prescribing before and after the VHA OSI initiative. Our hypothesis is that with guidance limiting or decreasing use of opioid therapy, clinicians may increase prescribing of non-opioid analgesics. To determine if such changes in published analgesic policies and initiatives indirectly affected patient outcomes, concurrent pain intensity ratings will be evaluated. 


\section{Methods}

\subsection{Study Design and Data Sources}

We conducted a retrospective, interrupted time-series, segmented regression model of aggregated monthly national data available from the VHA Corporate Data Warehouse (CDW), a national repository of patient-level medical records.[17] Data for this investigation were part of an observational study evaluating the safety and effectiveness of analgesics in older veterans with arthritis of the knee or hip. Outpatient prescription and pain intensity ratings and demographic covariate data from VHA CDW files[17] included name of analgesic and prescription release date, as well as any documented pain intensity ratings. Data was then aggregated monthly over a five-year study period and prescribing rates and summary pain intensity ratings calculated for entry into segmented regression models controlled for sociodemographic characteristics. Study protocols were approved by the VA Central Institutional Review Board (VA Central IRB Study 13-31, 1 I01 HX000911-01A2, IIR 12-106).

\subsection{Veterans Health Administration (VHA) Opioid Safety Initiative}

To study the overall trends in analgesic prescribing and the impact of a guideline-based analgesic safety initiative, total number of analgesic prescriptions and pain intensity ratings were evaluated in the 2 years prior to and 3 years after the VHA OSI. The OSI was a national VHA clinical initiative to promote safe opioid-related prescribing that completed rollout to all VHA facilities in October 2013.[9; 18]

\subsection{Subjects}

A national sample of all VHA patients $\geq 50$ years of age from January 1, 2012 - December 31, 2016, diagnosed with osteoarthritis of the knee and/or hip: ICD9: 715.15, 715.16, 715.25,715.26, 715.35, 715.36, 715.95, 715.96; ICD10: M16.0, M16.1, M16.10, M16.11, M16.12, M16.2, M16.3, M16.30, M16.31, M16.32, M16.4, M16.5, M16.50, M16.51, M16.52, M16.6, M16.7, M16.9, M17, M17.0, M17.1, M17.10, M17.11, M17.12, M17.2, M17.3, M17.30, M17.31, M17.32, M17.4, M17.5, M17.9, M13.15, M13.151, M13.152, M13.159, M13.16, M13.161, M13.162, M13.169, M13.85, M13.851, M13.852, M13.859, M13.86, M13.861, M13.862, M13.869) was identified. Two outpatient visits or one inpatient encounter noting these ICD codes were required for inclusion. Sociodemographic variables included age (in years), gender, and race (white or non-white). Rates used for outcomes and covariates are calculated as the monthly outcomes or covariates (numerator) divided by the number of unique veterans (denominator) in a given month.

\subsection{Outcomes}

Monthly rates of musculoskeletal analgesic prescriptions. All outpatient analgesic prescriptions for a 5year period (January 1, 2012 - December 31, 2016) were recorded and aggregated as counts by study month. Musculoskeletal analgesics were categorized as: opioids, NSAIDs, acetaminophen, and other study analgesic prescriptions. The denominator for the calculation of prescription rates was the number of unique patients $\geq 50$ years of age, diagnosed with osteoarthritis of the knee or hip in a given month during the study period. Outcomes were adjusted for sociodemographic covariates, age, gender, and race.

Opioids were defined as all opioid agonists including tramadol and their fixed non-opioid combinations with a few exceptions. Excluded were opioid and non-opioid cough preparations. NSAIDs included 
celecoxib, diclofenac, etodolac, ibuprofen, indomethacin, ketoprofen, ketorolac, meloxicam, naproxen, piroxicam, salicylate, sulindac, and tolmetin. NSAIDs and acetaminophen were counted as separate prescriptions when used as single agents but not when in combination with opioids. Other study analgesics included menthol with and without salicylate, capsaicin, and local anesthetics.

Pain intensity ratings are collected as part of routine outpatient clinical care in the VHA. This is usually documented as "the presence and intensity of pain" with responses provided by veterans in response to the question, "Please rate your pain right now on a 0 (no pain) to 10 (worst pain imaginable) scale. Following recommendations for optimal distributions and best fitting models when using pain score data, we followed recommendations by Goulet et.al.[4] Only non-missing pain intensity ratings were included in analyses (i.e., missing was not counted as no or "zero" pain. Outpatient pain intensity ratings (excluding those from inpatient encounters) during the same study period were collected and summarized following recommended calculations for optimal model fit for zero-inflated Poisson distributions[4]: percentage of those reporting pain ( $0=$ no pain or $1=a n y$ pain), and the mean pain intensity rating of those reporting pain (rating, range 1-10 highest).

\subsection{Analysis}

Analyses followed a similar approach to that of Lin et.al. that modeled VHA high-dose opioid therapy and concurrent benzodiazepine prescriptions $[9 ; 19]$ : segmented regression analyses of interrupted time series.

All variables were examined for missing values and for appropriate distributions and summarized as mean and standard deviations for continuous and percentages for categorical measures.

For the evaluation of pain intensity ratings, only outpatient ratings were used. A total of 10,350,959 separate outpatient pain intensity ratings over the 5 -year period were summarized as monthly values: proportion of those reporting pain ( 0 v. 1-10) and mean of those reporting pain (1-10 scale). Pain intensity ratings were also summarized as percent reporting mild (1-3), moderate (4-6), and severe (710) pain. As common to all observational studies involving pain ratings, participants having more pain would more likely receive both stronger medication as well as more frequent appointments and assessments of pain intensity. However, these are recognized limitations of using observational data. Distributions were compared to the results of previous VHA system-wide analysis of pain score distributions[4] to validate the consistency of pain intensity ratings over time.

The sample sociodemographic characteristics were examined. Raw counts for each category of study analgesic prescriptions (Total Study Analgesics, Opioid, NSAID, Other Study Analgesics, and Acetaminophen) were computed for each month. These counts were standardized by dividing by the number of days in each month and then multiplied by the length of an average month (365.25/12=30.44 days) and rounded to a whole number. Raw and standardized counts were examined and graphed. Finally, rates were calculated as follows: the number of prescriptions of each analgesic type within a standard month was divided by the number of unique patients (50+ years of age and meeting the diagnostic criteria) for the entire VHA within that month and multiplied by 100 to achieve a standardized monthly rate in mean prescriptions per 100 person-months. 
For analgesic prescribing, Poisson regression was performed to model the standardized monthly prescription count (offset by the log [number of unique patients in that month]). All models exhibited highly significant over-dispersion and required negative binomial regression methods.

Subsequently, negative binomial regression models were performed with the following variables: sociodemographic covariates (mean age [years], gender [\% male], race [\% white]); time (in months) for overall model linear slope; an indicator variable for OSI (pre or post) or step; and an interaction term between time and the indicator variable for OSI implementation to assess the possible change in slope $(\Delta \beta)$. A significant step term would signify a sharp increase or decrease - a step change - in prescribing associated with the OSI. Of note, the changes in slope (interaction term), slope change, $(\Delta \beta)$ from the pre- to post-OSI periods are of more substantive interest in demonstrating the effect of the OSI intervention.

Autocorrelation was investigated using several methods (harmonic terms and ACF/PACF graphs). Because of the lack of consistent autocorrelation, we ultimately decided to use Poisson-family models that resulted in best fit with negative-binomial models (see below response) with no adjustments for autocorrelation/seasonality.

Finally, pain intensity ratings were analyzed similarly with covariate-adjusted linear regression. Because pain intensity ratings typically conform to a zero-inflated Poisson, negative binomial distribution, or a hurdle Poisson/negative binomial distribution[4], the monthly average ratings were summarized by two complementary methods: proportion reporting pain (1-10 v. 0), and mean pain intensity rating for those reporting pain (1-10). All models were additionally adjusted for the same three covariates: mean age (years), gender (\% male), race (\% white). As with the negative binomial models, a step change would indicate an increase or decrease in pain intensity ratings associated with the OSI, while significant interaction term or change in slope would signal an increasing or decreasing trend in the level of pain post-OSI compared to pre-OSI. In addition, the percent of mild (1-3), moderate (4-6), and severe (7-10) pain were analyzed and graphed as described above, multivariable linear regression.

Effect sizes for each of the prescription and pain intensity models were determined as follows. The predicted values and 95\% confidence intervals for the end of the study (month 60, December 2016) was calculated from both the pre-OSI model and the full (pre-/post-OSI) model. These separate estimates were used to calculate the percent difference between the two prediction points with the pre-OSI prediction as the reference. A contrast was then performed for the pre/post-OSI model to decide significance (Hochberg adjusted) of these differences. The resulting predicted lines for both models were graphed along with unadjusted rates for comparison.

\subsection{Sample Size and Probability Adjustments}

Since the analyses involve counts in the thousands and denominators in the hundreds of thousands, like many previous studies of national databases, we did not perform an a priori sample size assessment [9]. Albeit, these aggregate summary measures (rates and means) comprise the datapoints for the analyses. We employed a quasi-experimental design (segmented regression of interrupted time series), in which it is generally accepted that more than a dozen points on either side of the "event"/inflection point of interest are enough to provide stable slope estimates. 
Nevertheless, for the negative binomial sample size calculation, we performed a post hoc calculation for the total prescription rates results using $R$ package, power.nb.test, showing that indeed, 12 values on each side of the inflection point is sufficient for $80 \%$ power ( $m u 0=26.6$, mu1=33.0, RR=1.011 (pre-/postOSI), theta=632, duration=1, $\alpha=0.05 /$ two-sided). We had 22 pre-OSI and 38 post-OSI values.

For the linear regression models, we used $G^{*}$ Power version 3.1.9.2 for overall $R^{2}$ : Multiple linear regression using $\alpha=0.05$, Power $=0.80, \mathrm{n}$ predictors $=6$, and effect size $=.35$ (large), a sample size of 46 (data points) is required. $\mathrm{N}=60$ for our analysis. The range of effect sizes we saw for the linear regression $\left(R^{2}\right)$ was $0.29-0.91$ (Table 2 ).

Hochberg adjustments were applied to the resulting probabilities within the estimates for the negative binomial models and within the linear regression models.

All analyses were performed with RStudio (Version 1.0.153, Boston, MA, USA) using R Version 3.4.2 and SAS version 9.4 (CSAS Institute, Cary, NC, USA). 


\section{Results}

During the study period, there were a total of 8,384,564 prescriptions written for 348,787 unique patients that met inclusion criteria (Table 1). Mean age for this cohort was 63.4 years (SD, 8.6) ranging from 50-104 years. Mean age for the cohort increased slightly over the study period from 64.3 to 66.3 years ( $\beta=0.03, p<.0001$ - not shown). Percent male gender (mean, 93.3\%; range 92.4-93.6; $\beta=-0.02, p$ $<.0001$ - not shown) and percent white race (mean, 69.2\%; range 67.9-70.7; $\beta=-0.04, p<.0001-$ not shown) demonstrated small but significant decreases over the same period. The denominator for the monthly calculation of prescription rates was all veterans over 50 years old with the diagnosis of osteoarthritis of the knee or hip, including those who did not receive any analgesic prescriptions, in the study period (overall, 499,243 unique patients).

\subsection{Study Analgesics}

Total Study Analgesics (Tables 2 \& 3, Figure 1): For total study analgesic prescriptions, there was a positive (increasing) trend pre-OSI ( $\beta_{\text {time }}=0.012, p=0.003$ ). At the initiation of the OSI there was a significant step $(0.319, p<.0001)$ as well as a pronounced negative change in slope $(\Delta \beta=-0.010, p<.0001)$. The post-OSI slope was not significantly different from zero or a flat trajectory. Total study analgesic prescriptions increased until the OSI inflection point, after which it displayed a flat trajectory (Figure 2). The effect size demonstrated a $30.6 \%(\mathrm{p}<.001)$ drop in total analgesic prescribing compared to what would have been predicted (estimate, 95\% Cl) under the pre-OSI trend, 48.1 (41.9-55.2) v. 33.4 (31.335.7) prescriptions/100 person-months.

Opioids (Tables 2 \& 3, Figure 1): Pre-OSI there was an increase in opioid prescribing $\left(\beta_{\text {time }}=0.018\right.$, $p<.0001$ ) along with an increase in prescribing at about the time of the OSI (step change: $0.578, p$ $<.0001)$. These trends were followed by a negative $\Delta \beta(-0.019, \mathrm{p}<.0001)$. Figure 2 shows the pre-OSI increase in opioid prescribing followed by a pronounced sustained decrease post-OSI. Extrapolating the pre-OSI trend would predict 32.4 (28.3-37.1) prescriptions/100 person-months compared to 16.9 (15.718.2 ) predicted under the full model (post-OSI), a significant $-47.8 \%$ change $(p<.001)$.

NSAIDs (Tables $2 \& 3$, Figure 2): NSAIDs prescriptions were stable over the study period $\left(\beta_{\text {time }}=0.002\right.$, $p=0.743$ ) showing no change in either step or slope change in the post-OSI period $(\Delta \beta=-0.001, p=0.743)$. Figure 3 illustrates an unchanging non-significant increase in NSAID prescribing over the entire study period without any associated perturbation related to the OSI. Similarly, the predicted lines from both the pre-OSI model and the full model show considerable overlap and no significant effect size changes, pre-OSI trend 10.7 (9.3-12.4) v. post-OSI trend 10.3 (9.7-11.0) with overlapping 95\% confidence intervals and a small relative effect size $(-3.7 \%, p=0.748)$.

Other Study Analgesics (Tables 2 \& 3, Figure 2): Other study analgesic prescriptions, increased modestly over the study period $\left(\beta_{\text {time }}=0.012, p<0.0002\right)$. However, there was no step change $(0.029, p=0.743)$ or change in slope in the post-OSI period $(\Delta \beta=-0.001, p=0.743)$. Other study analgesics showed increasing prescribing with no peri-OSI changes. End of study predictions resulted in only a $-2.3 \%$ and nonsignificant difference $(p=0.748)$.

Acetaminophen (Tables $2 \& 3$, Figure 2): Acetaminophen prescriptions demonstrated a flat trajectory over the study period $\left(\beta_{\text {time }}=0.006, p=0.255\right)$. Although a small and modestly significant step decrease was noted (step $=-0.145, p=0.004$ ) and a very modest significant increase in slope in the post-OSI period 
was noted $(\Delta \beta=0.005, p=0.047)$. The effect size calculation mirrored these results with modestly significant $(10.5 \%, p=0.003)$ increase in acetaminophen prescribing at the end of the study compared to the pre-OSI trend, post-OSI: $2.1(2.0-2.3) \vee 1.9(1.6-2.2)$ prescriptions/100 person-months, respectively.

\subsection{Pain Intensity Measures}

Figure 4 illustrates the pain intensity measures and their trajectories related to OSI implementation over the study period.

Pain Intensity in those Reporting Pain (Tables $2 \& 3$, Figure 1): The slope of pain intensity in those reporting pain was unchanged over the study period $\left(\beta_{\text {time }}=0.003, p=0.613\right.$ ) and showed no changes related to OSI (step=0.015, $p=0.963 ; \Delta \beta=0.000, p=0.963$ ). Effect size calculations showed a nonsignificant $0.1 \%(p=0.870)$ increase in Pain Intensity in those reporting pain between the pre- and pre/post (full)-models.

Percentage Reporting Pain (Tables 2 \& 3, Figure 3): The percentage reporting pain increased gradually throughout the study period ( $\beta_{\text {time }}=0.089, p, p=0.038$ ). As with the other pain intensity measures (Figures $1 \& 3)$, there were no step $(0.926, p=0.271)$ or slope changes $(\Delta \beta=-0.029, p=0.662)$ associated with OSI. The effect size calculation for the end of study was non-significant $(-1.9 \%, p=0.096)$.

Percent Reporting Mild, Moderate, and Severe Pain (Tables 2 \& 3, Figure 3): Breaking those Reporting Pain into Mild (1-3), Moderate (4-6), and Severe (7-10) pain revealed that there was a significant steady upward trend in those reporting Moderate Pain $\left(\beta_{\text {time }}=0.046, p=0.047\right)$. Mild pain exhibited a small, nonsignificant decrease over the study period $(-0.016, p=0.729)$. Severe pain showed no significant slope over the study $(0.059, p=0.109)$. The changes associated with OSI were small (all $<3 \%$ change), with no measure demonstrating any significant change associated with OSI (i.e., no significant step, $\Delta \beta$, or effect size change). 


\section{Discussion}

\subsection{Opioid and Non-opioid Analgesics}

This study described covariate-adjusted outpatient analgesic trends (opioid prescribing, non-opioid analgesic prescribing, and pain intensity ratings), to understand more comprehensively prescribing trends for an older group of OA patients before and after implementation of the VHA Opioid Safety Initiative.

We found a rise in total analgesic prescriptions pre-OSI, driven primarily by increasing opioid prescriptions with lesser contributions from other study analgesics and acetaminophen. In the post-OSI era, there was a dramatic reduction in overall analgesic prescribing. This trend occurred because of the pronounced decrease in opioid prescribing in the post-OSI period which was partially compensated by increased prescriptions from the other categories, a general increase in other study analgesics prescribing over the entire study period and a modest increase in acetaminophen prescribing post-OSI. NSAID prescribing appeared to continue unchanged through the study period.

The changes in these analgesic prescribing patterns did not parallel changes in overall reported pain intensity in this sample. Covariate-adjusted pain intensity measures (percentage reporting pain, but not pain intensity ratings in those reporting pain) show a clinically small, but significant steady increase over the entire study period. This was apparently due to a growing number of those experiencing moderate pain over the entire study period, compared to mild or severe pain. No step or slope changes associated with OSI were seen with any pain intensity measures.

Previous studies have demonstrated inflection points $[6 ; 9 ; 16]$ with opioid prescribing, albeit at different times. Kazanis et al.,[6] for example, using time-series forecasting models with both military and civilian data showed increasing and then decreasing prescriptions for opioids (inflection point about 2011)[6]. We did not find a decrease in the 2011-2013 period as they did. Other studies have demonstrated that educational programs, state monitoring programs, and a "Best practices initiative" continue to be effective in reducing opioid prescribing.[16] Our findings mirrored those of Lin et al., 2017[9] using VHA data demonstrating the increase and subsequent decrease in opioid prescribing prepost the 2013 OSI respectively.

As the opioid epidemic continues to be a public health threat with risk of adverse outcomes from prescription opioids, clinicians will increasingly be challenged to balance adequately treating patient pain while limiting the use of opioid analgesics. As previously mentioned, other studies indict and challenge the benefits of opioids in various disease states, including osteoarthritis. [7] Findings from this study demonstrate that with programs to ensure safer opioid prescribing practices, there has been an effective reduction in the use of opioids but also a concurrent increase in the use of non-opioid analgesic alternative medications. . Acetaminophen showed a very modest increase in prescribing post-OSI. There continues to be a steady increase in other study analgesic use, but not one that is at a greater rate of increase than prior to the OSI. If these practice trends continue, there should be awareness and monitoring of the impact that the greater use these analgesic medications may have on safety, effectiveness, and clinical outcomes for the patients being treated.[10; 21] Clinicians will need to be informed of the risks and benefits of these analgesic alternates in older patients. Future studies should evaluate the safety and effectiveness of not only opioid analgesic use, but also these alternate non- 
opioid analgesic medications that appear to be increasingly used, as indicated by this study, to treat pain in older persons.

\subsection{Limitations}

This study has several limitations. Data and results do not reflect causality as this was an observational study. In addition, findings from VHA data, particularly in this older subset, may not be generalizable to other health care settings and patient populations. While the emphasis was on evaluation of prescribing trends before and after OSI implementation, undoubtedly, concurrent national policies related to analgesic prescribing, as well as public awareness of risks, may have also impacted changes in prescribing trends.

In this investigation, data on non-VHA prescribed medications and self-administration of over-thecounter medications or alternative therapies were not available. We did not evaluate co-prescribing of sedative medications nor calculate the doses of prescribed opioids (as in morphine equivalent doses) but confined our analyses to prescription rates. Lastly, the presence of co-morbid medical (such as cancer or other terminal) and mental health conditions was assumed to be randomly distributed and stable over the study period.

In the future, an interrupted time-series series analysis will be used to investigate seasonality, produce forecasts to compare with subsequent data, as well as execute a more comprehensive model to associate all the other non-opioid analgesic medications with opioid trends.

\subsection{Summary}

In conclusion, recent trends and opioid prescribing safety initiatives have been effective and as demonstrated by this and other studies; there are decreasing rates of opioid prescribing for older veterans with osteoarthritis of the knee and hip. These changes in decreased opioid prescribing are largely not compensated by the prescribing of other analgesic classes in these older patients. However, changes in analgesic prescribing do not appear to be associated with concurrent changes in reported pain intensity by older veterans with osteoarthritis. Future studies should investigate potential risks and benefits of these changing rates of opioid and non-opioid analgesic medications and the impact these have on patient safety and pain intensity outcomes. 


\section{Acknowledgements:}

The EAASE (Evaluating Arthritis Analgesic Safety and Effectiveness) team includes: Ruth Balk, Rachel Dismore, Vera Gaetano, Dorian Gittleman, David Leverty, Erin Linden, Brittany Majeski, Ebony Manuel, Kai Monde, Diana Natividad, Grace Polusny, Anthony Rinaldi, Daniel Signor, Lee Stefanis.

\section{Conflicts of Interest:}

There are no conflicts of interest for authors. This work was supported by Award Number 1101 HX000911-01A2 from the Health Services Research \& Development Service of the VA Office of Research and Development. The contents of this paper are solely the responsibility of the authors and do not represent the views of the U.S. Department of Veterans Affairs or the United States Government. 
Tables and Figures

Figure Legends:

Figure 1. EAASE National Cohort, Rates for All Study Analgesic Prescriptions (2012-2017). Opioid and non-opioid prescription classes and total analgesic prescriptions as rates (mean count/100 person-months). Counts were adjusted according to the length of a standard month (365.25 days $/ 12=30.44$ days) to account for the varying length of each month.

Figure 2. EAASE National Cohort, Rates for Total Analgesic Prescriptions, Opioid Prescriptions, and Mean Pain Intensity Score for Those Reporting Pain (2012-2017). Rates and superimposed lines from multivariable negative binomial models for prescription rates and a multivariable linear regression model for Mean Pain Intensity including terms for slope, step change (pre-/post-OSI), and change in slope. Pre-OSI models and predicted values (dashed lines) include only term for slope. All models additionally adjusted for age (years), male gender (\%), and white race (\%).

Figure 3. EAASE National Cohort, Rates for Nonsteroidal Anti-inflammatory Drugs (NSAIDs), Other Study Analgesics and Acetaminophen (2012-2017). Rates and superimposed lines from multivariable negative binomial models including terms for slope, step change (pre-/post-OSI), and change in slope. Pre-OSI models and predicted values (dashed lines) include only term for slope. All models additionally adjusted for age (years), male gender (\%), and white race (\%).

Figure 4. EAASE National Cohort, Percent values for Pain Intensity Measures: Percentages Reporting Pain, Mild Pain (1-3), Moderate Pain (4-6), and Severe Pain (7-10) (2012-2017). Monthly mean percentages and superimposed lines from multivariable linear regression models including terms for slope, step change (pre-/post-OSI), and change in slope. Pre-OSI models and predicted values (dashed lines) include only term for slope. All models additionally adjusted for age (years), male gender (\%), and white race (\%). 
References:

[1] Ackerman IN, Zomer E, Gilmartin-Thomas JF, Liew D. Forecasting the future burden of opioids for osteoarthritis. Osteoarthr Cartil 2018;26:350-355.

[2] Bohnert AS, Valenstein M, Bair MJ, Ganoczy D, McCarthy JF, Ilgen MA, Blow FC. Association between opioid prescribing patterns and opioid overdose-related deaths. JAMA 2011;305:1315-1321.

[3] Compton WM, Boyle M, Wargo E. Prescription opioid abuse: problems and responses. Pain Med 2015;80:5-9.

[4] Goulet JL, Buta E, Bathulapali H, Gueorguieva R, Brandt CA. Statistical models for the analysis of zeroInflated pain intensity numeric rating scale data. J Pain 2017;18(3):340-348.

[5] Hargan ED. Determination that a public health emergency exists. In: Department of Health and Human Services editor. Washington D.C., October 26, 2017.

[6] Kazanis W, Pugh MJ, Tami C, Maddry JK, Bebarta VS, Finley EP, McGeary DD, Carnahan DH, Potter JS. Opioid use patterns among active duty service members and civilians: 2006-2014. Mil Med 2018;183:e157-e164.

[7] Krebs EE, Gravely A, Nugent S, Jensen AC, DeRonne B, Goldsmith ES, Kroenke K, Bair MJ, Noorbaloochi S. Effect of opioid vs. nonopioid medications on pain-related function in patients with chronic back pain or hip or knee osteoarthritis pain: The SPACE Randomized Clinical Trial. JAMA 2018;319(9):872-882.

[8] LaFrenais FL, Bedder R, Vicerstaff V, Stone P, Sampson EL. Temporal trends in analgesic use in longterm care facilities: a systematic review of international prescribing. J Am Geriatr Soc 2018;66:376-382.

[9] Lin LA, Bohnert AS, Kerns RD, Clay MA, Ganoczy D, Ilgen MA. Impact of the Opioids Safety Initiative on opioid-related prescribing in veterans. Pain 2017;158(5):833-839.

[10] Machado GC, Maher CG, Ferreira PH, Pinheiro MB, Lin CC, Day RO, McLachlan AJ, Ferreira ML. Efficacy and safety of paracetamol for spinal pain and osteoarthritis: systematic review and meta-analysis of randomised placebo controlled trials. BMJ 2015;350:h1225.

[11] Mosher HJ, Krebs EE, Carrel M, Kaboli PJ, Vander Weg MW, Lund BC. Trends in prevalent and incident opioid receipt: an observational study in Veterans Health Administration 2004-2012. J Gen Intern Med 2014;30(5):597-604.

[12] Office of the Assistant Secretary for Planning and Evaluation. Opioid abuse in the U.S. and HHS actions to address opioid-drug related overdoses and deaths., U.S. Department of Health \& Human Services. ASPE Issue Brief. Washington D.C., 2015. Available at file://C:/Users/trent/Documents/VA\%20Research/EAASE_TS/Pain\%20Journal/Bibliography/ib_ Opioidlnitiative.pdf. Accessed 10/10/2018.

[13] Patrick SW, Fry CD, Jones TF, Buntin MB. Implementation of prescription drug monitoring programs associated with reduction in opioid-related death rates. Health Aff (Millwood) 2016;35(7):13241332.

[14] Paulozzi L, Rudd RA, Jones CM, Mack KA. Vital signs: Overdoses of prescription opioid pain relievers - United States, 1999-2008. Morb Mortal Wkly Rep 2011;60(43):1487-1492.

[15] Rudd RA, Aleshire N, Zibbel JE, Gladden RM. Increases in drug and opioid overdose deaths - United States, 2000-2014. Morb Mortal Wkly Rep 2016;60(43):1487-1492.

[16] Sun BC, Lupulescu-Mann N, Charlesworth CJ, Kim H, Hartung DM, Deyo RA, John McConnell K. Impact of hospital "Best Practice" mandates on prescription opioid dispensing after an emergency department visit. Acad Emerg Med 2017;24:905-913.

[17] VA Informatics and Computing Infrastructure (VINCI). Corporate Data Warehouse (CDW), Vol. 2018, 2018.

[18] VHA Pain Management. Opioid Safety Initiative (OSI), Vol. 2018, 2018. 
[19] Wagner AK, Soumerai SB, Zhang F, Ross-Degnan D. Segmented regression analysis of interrupted time series studies in medication use research. J Clin Pharm Therap 2002;27:299-309.

[20] Weiner SG, Baker O, Poon SJ, Rodger AF, Garner C, Nelson LS, Schuur JD. The effect of opioid prescribing guidelines on prescriptions by emergency physicians in Ohio. Ann Emerg Med 2017;70(6):799-808.

[21] Wongrakpanich S, Wongrakpanich A, Melhado K, Rangaswami J. A comprehensive review of nonsteroidal anti-inflammatory drug use in the elderly. Aging Dis 2018;9(1):143-150. 